\title{
Do Retail Clinics Increase Early Return Visits for Pediatric Patients?
}

\author{
James E. Rohrer, PhD, Kathleen M. Yapuncich, MD, Steven C. Adamson, MD, and \\ Kurt B. Angstman, MD
}

Objective: The purpose of this study was to assess the risk of early return visits for pediatric patients using a retail clinic.

Methods: We used medical records of pediatric patients seen in a large group practice in Minnesota in the first 2 months of 2008. A retrospective analysis of electronic patient records was performed on 2 groups of patients: those using the retail clinic $(n=200)$ and a comparison group using a same-day acute family medicine clinic in a medical office $(n=200)$. Two measures of early return visits were used as dependent variables: office visits within 2 weeks for any reason and office visits within 2 weeks for the same reason. Multiple logistic regression analysis was used to adjust for case mix differences between groups. Trained medical records abstractors reviewed electronic medical records to obtain the data.

Results: After adjustment for baseline differences in age, acuity, and number of office visits in the previous 6 months, no significant differences in risk of early return visits were found among clinic types.

Conclusions: Retail clinic visits were not associated with early return visits. (J Am Board Fam Med 2008;21:475-476.)

Retail clinics are more convenient than scheduled appointments for low acuity office visits. On the other hand, retail clinics may increase the cost of medical care if they are more likely to result in an early return visit. The purpose of the our study was to test the hypothesis that visits to a newly opened retail clinic were more likely than same-day acute visits to be followed by an office visit within 2 weeks.

In this retrospective cohort study of medical records, the first 200 consecutive patients (age $<18$ ) seen in a new retail clinic during its first 3 weeks of operation (late January and early February of 2008) were regarded as the exposed group. The comparison group was 200 consecutive patient vis-

This article was externally peer reviewed.

Submitted 11 April 2008; revised 1 May 2008; accepted 9 May 2008.

From the Department of Family Medicine (JER, SCA, KBA) and Department of Pediatrics (KMY), Mayo-Clinic Rochester, Minnesota.

Funding: none.

Conflict of interest: none declared.

Corresponding author: James E. Rohrer, PhD, Department of Family Medicine, Mayo Clinic, 200 First Street SW, Rochester, MN 55905 (E-mail: rohrer.james@mayo.edu). its provided in the same-day acute clinic at a downtown clinic location.

Patients in the retail clinic were older (mean 7.5 vs $6.2, P<.01)$ and had used fewer office visits in the previous visit 6 months (median 2.0 vs $2.5, P<$ $.01)$.

Thirty-one $(15.5 \%)$ of retail clinic patients received an office visit within 2 weeks (to any location for any reason), and 51 (25.5\%) of same-day acute visitors returned $(P=.01)$. Number of return office visits for the same reason for retail clinic patients and same-date acute visitors were $13(6.5 \%)$ and 25 (12.5\%), respectively $(P<.04)$. Although these bivariate comparisons were significant, they were not confirmed after adjusting for group differences using multiple logistic regression analysis (Table 1). In the analysis of return visits for any reason, the adjusted odds ratio for same-day, acute clinic patients versus retail clinic patients was 1.62 (CI, $0.96-2.73)$. In the analysis of return visits for the same reason, the adjusted odds ratio for same-day, acute clinic patients versus retail clinic patients was 1.79 (CI, 0.87 to 3.69). However, the number of office visits in the previous 6 months was significant in both models; in the analysis of return visits for 
Table 1. Multiple Logistic Regression Analysis of Return Office Visits $(n=400) *$

\begin{tabular}{lll}
\hline Variable & Odds Ratio & Confidence Interval \\
\hline Return visits for any reason & & \\
$\quad$ Group & 1.0 & 0.96 to 2.73 \\
$\quad$ Retail clinic & 1.62 & 1.08 to 1.25 \\
$\quad$ Same-day acute & 1.16 & 0.20 to 1.22 \\
Office visits in the previous 6 months & 0.49 & 0.98 to 1.09 \\
Acute visit (yes vs no) & 1.04 & \\
Age & & 0.87 to 3.69 \\
Return visits for same reason & & 1.02 to 1.17 \\
Group & 1.0 & 0.16 to 1.58 \\
$\quad$ Retail clinic & 1.78 & 0.93 to 1.07 \\
$\quad$ Same-day acute & 1.09 & 0.49 \\
Office visits in the previous 6 months & 1.00 & \\
Acute visit (yes vs no) & & \\
Age &
\end{tabular}

*All included variables are shown in the table.

any reason, the odds ratio was $1.16(\mathrm{CI}, 1.08$ to $1.25)$, and in the analysis of return visits for the same reason, the odds ratio was 1.09 (CI, 1.02 to 1.17).

The American Academy of Pediatrics opposes retail-based clinics because of its commitment to the medical home model. ${ }^{1}$ Retail clinics potentially can fragment care, miss opportunities for preventive services, lack appropriate education of patients and parents, and fail to provide appropriate follow up.

On the other hand, retail clinics eliminate the need for appointments, solve the parking problem that is so common in downtown clinics, and allow parents to combine shopping with clinic visits. Retail clinics offer lower cost per visit because the clinics are staffed by nurse practitioners and physi- cians assistants rather than by physicians. Physicians can be reached as needed by telephone, but this form of medical supervision incurs minimal additional cost.

Retail convenience clinics are not intended to serve as the sole source of care. The external validity of this study is limited because it only examined one retail clinic. Results might differ with other retail clinics of different types, using different staff, and with different patient populations.

Data were abstracted by Kelly Amunrud and Julie Maxson.

\section{References}

1. Retail-Based Clinic Policy Work Group, AAP. AAP principles concerning retail-based clinics. Pediatrics 2006;118:2561-2. 